\title{
Superior vena cava resection for lung and mediastinal malignancies
}

\author{
Lorenzo Spaggiariab*, Francesco Petrellab ${ }^{\mathrm{b}}$, Francesco Leo ${ }^{\mathrm{b}}$, Giulia Veronesi ${ }^{\mathrm{b}}$, \\ Piergiorgio Sollib, Alessandro Borrib, Domenico Galettab, Roberto Gasparrib, \\ Paolo Scanagattab
}

\author{
a University of Milan School of Medicine, Milan, Italy \\ ${ }^{\mathrm{b}}$ European Institute of Oncology, Division of Thoracic Surgery, Via Ripamonti 435, 20141 Milan, \\ Italy
}

Even though the benefit of superior vena cava resection for lung and mediastinal malignancies remains controversial, the recent international experiences have demonstrated technical feasibility of such an extended surgery with acceptable postoperative morbidity and mortality. Concerning lung cancer, a multicentric international study over a 40 -year period reports a risk of developing postoperative complications and mortality of $30 \%$ and $12 \%$, respectively, with a 5 -year probability of survival of $21 \%$. The same study, analysing the results of the last 10 years, demonstrated an improvement of the outcome with a $6 \%$ of postoperative mortality, with a 5 -year probability of survival of $28 \%$. With regards to mediastinal malignancies, completeness of surgical resection is still considered one of the most important prognostic factors, and extended SVC resection could improve local control and disease free survival. In conclusion, radical resection of lung cancer and mediastinal malignancies involving SVC, is feasible, and it could lead to permanent cure in carefully selected patients.

Keywords: Extended resection; Lung cancer; Mediastinal tumour; Superior vena cava; Surgery

\section{Introduction}

In non-small cell lung cancer (NSCLC) and mediastinal malignancies, superior vena cava (SVC) infiltration was long considered an absolute contraindication to surgical resection [1]. The absence of suitable prosthetic material for SVC replacement, uncertainties regarding the effects of SVC clamping, the seemingly high probability of graft thrombosis and infection after lung resection, and last but not least, the dismal outcomes of pilot experiences, strongly discouraged surgical approaches. Nevertheless, work on animals and

\footnotetext{
* Corresponding author: Tel.: +39-02-5748 9666; fax: +39-0257489698.

E-mail: lorenzo.spaggiari@ieo.it
}

humans in the 1970s and 1980s demonstrated the technical feasibility of extended surgery for mediastinal and pulmonary malignancies, and long-term survival was achieved in some instances. To our knowledge, 11 series reporting more than six patients undergoing SVC replacement in the context of NSCLC have been published in the English literature over the last 15 years [1-12]. Postoperative mortality ranged from $0 \%$ to $22 \%$, with 5 -year survival between $21 \%$ and $31 \%$ (Table 1). In 2004, a multicentric retrospective study was published [10] analysing data on 109 patients. Major postoperative morbidity and mortality were $30 \%$ and $12 \%$, respectively, and fiveyear survival was $21 \%$ (28\% considering patients treated over the recent 10 years). These findings 
L. Spaggiari et al. / Multimedia Manual of Cardiothoracic Surgery / doi:10.1510/mmcts.2005.001511

Table 1. English-language literature review (1990-2005): full papers with more than 6 patients

\begin{tabular}{llcccc}
\hline Ref. & Author/year & No. of patients & Prosthetic graft & Mortality & 5-year survival \\
\hline$[2]$ & Piccione/1990 & 6 & 0 & $17 \%$ & NR \\
{$[3]$} & Thomas/1994 & 15 & 4 & $7 \%$ & $24 \%$ \\
{$[4]$} & Tsuchiya/1994 & 32 & 7 & $22 \%$ & NR \\
{$[5]$} & Dartevelle/1997 & 14 & 14 & $7 \%$ & $31 \%$ \\
{$[6]$} & Fukuse/1997 & 8 & 3 & NR & NR \\
{$[7]$} & Spaggiari/2000* & 25 & 7 & $12 \%$ & $29 \%$ \\
{$[8]$} & Spaggiari/2000** & 11 & 3 & 0 & NR \\
{$[9]$} & Bernard/2001 & 8 & 2 & NR & $25 \%$ \\
{$[10]$} & Spaggiari/2004 *** & 109 & 9 & $12 \%$ & $21 \%$ \\
{$[11]$} & Shargall/2004 & 15 & 11 & $14 \%$ & $57 \%(3$ years) \\
{$[12]$} & Suzuki/2004 & 40 & & $10 \%$ & $24 \%$ \\
\hline
\end{tabular}

* Marie Lannelongue Hospital, Paris, experience.

** European Institute of Oncology, Milan, experience.

*** Multicentric study [10].

showed that SVC surgery is technically feasible, and also that oncological outcomes can be better than in patients given chemotherapy and radiation only (fiveyear survival 5-17\%) [1].

For mediastinal cancers (thymomas, etc.) in particular, completeness of resection is a major prognostic factor. Thus, surgical techniques that allow the accomplishment of complete tumour resection, even in the presence of direct vascular invasion, are urgently required.

\section{Anatomy}

The right and left innominate veins, which receive venous blood mainly from the upper thorax, arms, neck and head, are the major vessels returning blood to the SVC. The SVC begins at the level of the first right costal cartilage and terminates in the right atrium at the level of the third intercostal space, and is thus located in the superior part of the middle mediastinum, to the right of the aorta, and anterior to the trachea and right main bronchus. The SVC is about $2 \mathrm{~cm}$ in diameter and $6-8 \mathrm{~cm}$ in length; the last $2 \mathrm{~cm}$ are within the pericardial reflection around the right atrium. The extrapericardial part of the SVC is surrounded by numerous lymph nodes.

A number of other veins drain into the SVC and can be major contributors to collateral flow and hence are important in SVC surgical anatomy.

Azygos and hemiazygos veins The azygos is the thoracic continuation of the right ascending lumbar vein; it collects blood from the right posterior intercostal veins and drains into the posterior SVC, just above the pericardial reflection. The hemiazygos vein is the continuation of the left ascending lumbar vein; it intercepts the lower left posterior intercostal veins, ascending on the left side of the thoracic spine as far as the eighth thoracic vertebral body, where it crosses over the vertebral column to fuse with the azygos vein.

Paravertebral system This is a low pressure circumferential venous network that has connections with the SVC and the inferior vena cava; it consists of an internal plexus within the spinal canal and an external plexus around the vertebral bodies, with anterior and posterior venous arches.

Lateral thoracic vein This runs vertically, draining into the axillary vein, which continues as the subclavian vein after passing under the first rib, and as the brachiocephalic vein after junction with the internal jugular vein.

Internal mammary (thoracic) vein This runs vertically behind the costal cartilages within the thoracic wall parallel to the sternum; it drains the anterior intercostal veins and superior epigastric vein, and itself drains into the subclavia.

Other pathways contributing to collateral flow are the jugular, thyroid, thymic and pericardium phrenic venous systems.

\section{History}

In 1757 William Hunter described a case of SVC syndrome caused by a syphilitic aneurysm of the ascending aorta [13]. In 1837 William Stokes reported the first case of SVC syndrome caused by a malignancy [14]. In 1949 Mclntire and Sykes reported the first series of 502 cases with SVC syndrome mainly caused by benign diseases such as syphilitic aortic aneurysm and chronic fibrous mediastinitis from 


\section{Spaggiari et al. / Multimedia Manual of Cardiothoracic Surgery / doi:10.1510/mmcts.2005.001511}

tuberculosis, only a third of the cases were due to primary thoracic cancers [15]. Prior to 1949, SVC syndrome had a mainly infectious etiology, now thoracic malignancies are the primary cause.

As regards surgery for SVC syndrome, in 1934 Carlson working on dogs found that SVC ligation below the azygos resulted in the death of all animals, while SVC ligation above the azygos allowed survival, demonstrating that the azygos system is an important collateral pathway [16]. The first successful bypass operations for SVC obstruction in humans were performed with autologous femoral vein grafts by Klassen in 1951 [17] and Bricker and McAfee in 1952 [18].

In 1961 Benvenuto and colleagues constructed large calibre bypass conduits from several segments of saphenous vein; these were incised longitudinally, flattened, placed over a stent in a panelled or tiled manner and sewn together to create the conduit [19].

In 1961 Schramel and Olinde described the subcutaneous tunnelling of a long saphenous vein bypass conduit to the jugular vein [20]. This technique was later adopted by Taylor and associates (1974) [21] and Vincze et al. (1982) [22] in seven patients with SVC obstruction due to lung cancer. In 1976 Doty and Baker performed the first successful venous bypass with a spiral saphenous vein graft [23]. This procedure had been developed two years previously by Chiu and associates who performed it in a patient with SVC obstruction secondary to granulomatous mediastinitis [24]. In 1986 Mitchell and colleagues described two SVC bypasses using intact saphenous vein in patients with mediastinal fibrosis [25].

In 1987 Dartevelle et al. described 13 patients with mediastinal or lung malignancies and SVC involvement: they were treated by SVC resection and reconstruction with polytetrafluoroethylene grafts [26].

\section{Pathology}

Lung cancer and thymoma are the malignancies that most commonly invade the vena cava. NSCLC can infiltrate the SVC in three ways:

1. Arising in the ventral segment of the right upper lobe, it may grow to invade the mediastinal pleura, the phrenic nerve and the anterolateral part of the SVC. The carina and trachea are often spared.

2. Arising in the central part of the main right bronchus, the cancer can directly invade the carina and SVC posteriorly at its confluence with the azygos. This type of invasion is often accompanied by R4 lymph node involvement, while the phrenic nerve
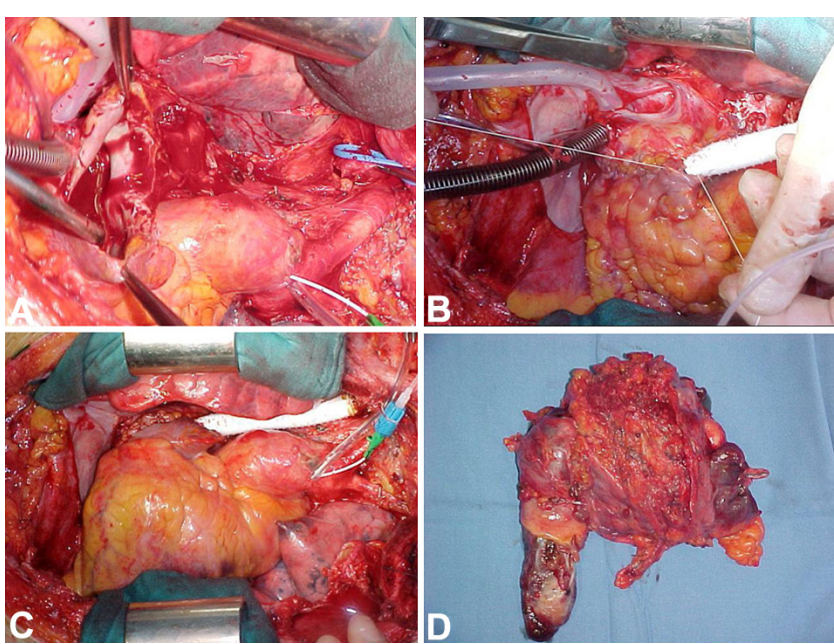

Photo 1. Patient with thymoma infiltrating SVC and neoplastic thrombus of right atrial cavity. (A) shows patient on cardiopulmonary bypass; atriotomy with resection of the SVC and partial resection of the right atrium. (B): construction of anastomosis between the right atrium and prosthesis. (C) shows completed anastomosis. Note the intracavity tumour in (D)

is often spared. In such cases the SVC can be resected en bloc with the carina.

3. The SVC is infiltrated from involved R4 lymph nodes.

Mediastinic cancers invading the SVC present differently. Thymomas, for example, usually grow slowly and asymptomatically and may become large before being diagnosed. Typically such cancers involve first the innominate veins and then the SVC. Resection, therefore, includes the SVC and its major divisions, and the type of reconstruction required differs from that employed for invasion from a lung. Because of the slow growth, one may occasionally observe complete neoplastic thrombosis of the SVC together with invasion of the atria (Photo $1 \mathrm{~A}-\mathrm{D}$ ) or multi-organ invasion (aorta, pulmonary arteries, etc.).

These differing habits determine the surgical access route and the type of vena cava and lung resection performed.

\section{Patient selection}

\section{Oncological selection}

A. Lung cancer. The workup of a patient with locally advanced lung cancer includes bronchoscopy to determine whether the carina is involved and if necessary plan enlarged bronchial tree resection. Whole body CT scan and PET scan are essential to determine whether distant metastases are present which would contraindicate surgery. If CT scan indicates operable disease, diagnostic cervical mediastinosco- 


\section{Spaggiari et al. / Multimedia Manual of Cardiothoracic Surgery / doi:10.1510/mmcts.2005.001511}

py is then required to exclude R2 node involvement (which would absolutely contraindicate surgery), and examine the tracheobronchial angle with a view to carina removal. The presence of (non bulky) R4 adenopathy is not an absolute contraindication to surgery as these nodes are so close to the tumour. A diagnosis of SVC infiltration is always clinical (cT4) as it is impossible to confirm blood vessel infiltration. After work-up is complete, the patient undergoes induction chemotherapy which has the following objectives:

- to extirpate systemic microscopic disease

- to identify rapidly growing disease which would contraindicate surgery

- to reduce disease extent in the mediastinum and facilitate resection. On completion of (usually three cycles of) chemotherapy, the patient is re-staged with total body CT, PET scan (bronchoscopy in case of tracheo-bronchial associated invasion).

B. Mediastinal tumours. Work-up in these patients includes total body CT scan, and PET scan. In cases of advanced disease it may be useful to perform MRI of the mediastinum to exclude aortic arch and its branches invasion. And in such patients diagnostic cervical mediastinoscopy may not be necessary because the invasion of the tracheo-bronchial tree is rare in these tumours, and the infrequent involvement of mediastinal lymph nodes is not a contraindication to surgical resection. If right atrial cavity involvement is suspected, echocardiogram (preferably transesophageal) may be useful. Induction chemotherapy is generally advisable in bulky mediastinal tumours.

Functional selection The work-up of lung cancer patients who are candidates for surgery must be complete and include functional evaluation. Spirometry, perfusion scintigraphy, echocardiography and exercise tolerance test must always be performed to ensure that cardiorespiratory reserve is adequate.

\section{Surgical procedure}

SVC removal requires total clamping which gives rise to acute intraoperative caval syndrome, whose severity will vary depending on the extent of pre-existing caval occlusion and consequent development of collateral circulation. Thus, if SVC involvement has not obstructed blood flow, complete clamping has major haemodynamic consequences; whereas if the lumen is compromised (as typically occurs with mediastinic tumours), complete clamping has much more modest repercussions for the pre-existing collateral circles; in fact it may not be necessary to reconstruct the SVC following its removal.
Acute intraoperative caval syndrome has several haemodynamic aspects: reduction of cardiac in/out put resulting in an increased venous head pressure and in reduced systemic arterial pressure. These events lead to a decrease in pressure gradient in the head resulting in marked tissue edema, particularly in the brain.

It is important to take steps to reduce these effects during surgery. This can be achieved (a) by cardiopulmonary bypass, (b) by inserting a temporary shunt [2] or (c) by means of the cross-clamping technique $[10,27]$. Techniques (a) and (b) are only used occasionally in SVC surgery. Cross-clamping is mainly used, and aims to maintain the vascular brain pressure gradient, so as to avoid edema. Increased venous pressure is contrasted with vasoactive fluids and drugs that increase arterial pressure. Rapid SVC substitution (with complete heparinisation) keeps the cross-clamping times as short as possible [27].

Cephalic venous pressure and systemic arterial pressure are monitored continuously during the operation; as are cardiac filling and haemodynamic changes during clamping (preferably by transesophageal ultrasonography). A double lumen catheter placed in the femoral veins allows one to obtain the vascular filling necessary during clamping.

Several surgical approaches are possible, and may depend on the personal preferences of the surgeon. The choice should, however, take into consideration the type of lung resection being performed, the size of the lesion, and whether or not the innominate veins are involved. A mediastinic cancer generally requires sternotomy or hemi-clamshell, or occasionally a clamshell approach. Lung cancer originating in the ventral segment of the superior lobe, but not involving the bronchus, can be approached by hemi-clamshell (see Video 3) or sternotomy; involvement of the bronchus or carina necessitates (in view of the successive reconstruction) a lateral or postero-lateral thoracotomy.

A partial or total SVC resection is performed depending on the extent of involvement. When less than 50\% of the circumference is infiltrated, a partial SVC resection is acceptable. Mechanical sutures (Photo 2), or continuous sutures after partial clamping may be used (Photo 3). Sometimes a patch reconstruction is required: heterologous or synthetic material may be used (Photo 4).

When more than $50 \%$ of the circumference is infiltrated a prosthesis is required. After a careful exploration of the chest cavity, SVC is dissected from mediastinal tissues and isolated with vessel loops 



Photo 2. (A-B) Partial resection of SVC by stapler after complete control of the vessel, but without clamping, for infiltration of azygoscaval confluence; (C) shows reduction in the final calibre of SVC.

(Videos 1-4), and then clamped using the crossclamping technique previously described (Videos 5 and 6). For a long time, the prostheses used were synthetic, typically PTFE graft (Photos 5-10) (Video 7).

Autologous materials have been also used (saphenous vein, pericardium etc.); recently we adopted a heterologous (bovine) pericardium graft made tubular during operation (Photos 11-13) (Videos 8-11).

Generally the prosthesis joins two ends of the SVC (Photos 5 and 6) (Videos 10 and 11). Sometimes the anastomosis may be between the left innominate vein and the SVC origin (Photo 9), removing the right venous trunk without reconstructing. Alternatively the anastomosis may be between the right innominate vein and the SVC, following total removal of the left innominate vein (Photos 13 and 14). Sometimes it is necessary to reconstruct the resected innominate vein (Photo 10B) or completely remove the left innominate vein and partially resect the SVC (Photo 15). Use of heparin $(0.5 \mathrm{mg} / \mathrm{kg})$ prevents thrombosis during prosthesis placement.

After SVC replacement, the tracheo-bronchial involvement (if associated), is removed and the airway reconstructed (Video 12).

Before discharge, the patency of the graft is studied by using both thoracic CT scan and phlebography; the follow-up of the graft, and of the oncological disease performed by periodical total body CT scan (Video 13).

\section{Results}

Two questions immediately arise with regard to SVC surgery: is it feasible? (is it reproducible with accept-


Photo 3. A similar case to that illustrated in Photo 2. Resection performed after partial SVC clamping. SVC reconstruction by a running polypropylene $5 / 0$ suture.

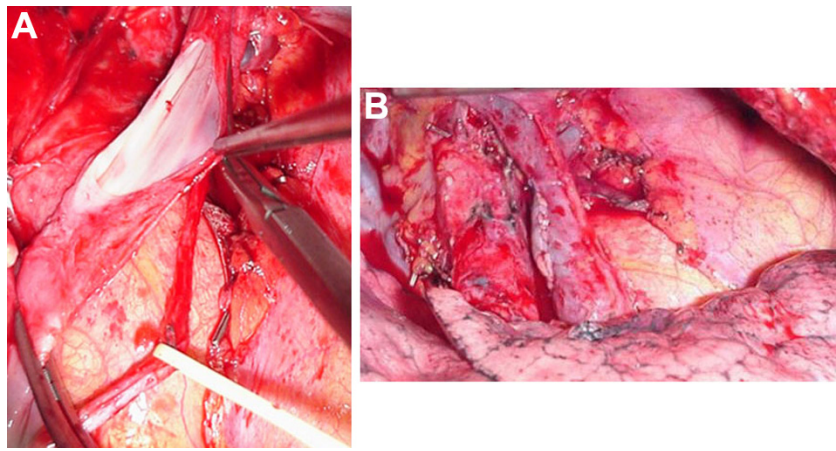

Photo 4. After partial SVC resection (A) a patch of autologous pericardium has been used to repair the defect (B). In this case the SVC clamping was complete.

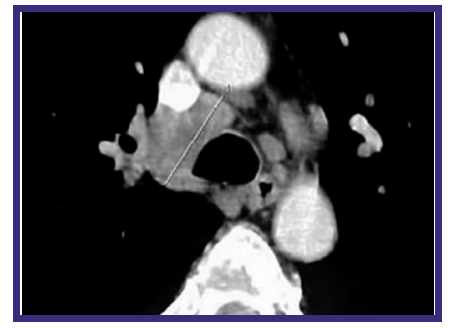

Video 1. (Patient A). This patient had NSCLC originating in the right main bronchus and infiltrating the carina. Mediastinoscopy was performed to exclude R2 nodal involvement. Induction chemotherapy was given. Given the need to remove the carina and the SVC, access was via lateral thoracotomy, sparing the muscle of the 5th intercostal space and section of the 4th costal cartilage. The operation proceeded initially with dissection of the SVC to achieve control of the vessel at its confluence with the innominate veins.

able morbidity and mortality?); and is it oncologically worthwhile? (Are results better than combination chemotherapy and radiotherapy?).

The technical feasibility of the operation was demonstrated in the 1970s and 1980s, supported by studies published in the 1990s. Postoperative complications and perioperative deaths (up to 30 days) were highly dependent on the surgeon's experience, type of SVC surgery performed (partial vs. complete SVC resection) and type of lung resection (lobectomy vs. tracheal sleeve pneumonectomy). 


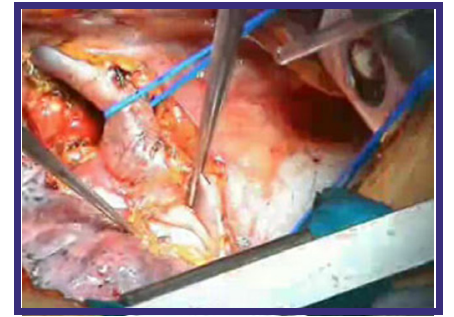

Video 2. (Patient A). The opening of the pericardium allows to control the SVC just before its entry into the right atrium. Dissection of the lymph nodes in Barety's space at this point favours control of the vessel. The right pulmonary artery is isolated within the Teile's sinus.

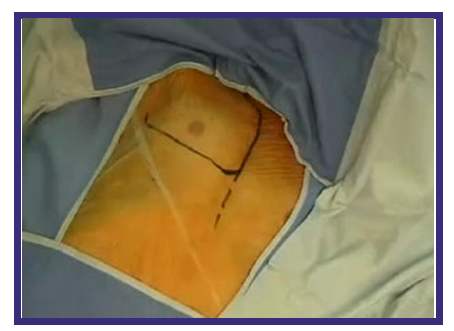

Video 3. (Patient B). This patient had lung cancer originating in the ventral part of the right superior lobe, without lymph node involvement by mediastinoscopy, and without bronchial tree involvement. A hemi-clamshell was used for access. The SVC was isolated after complete mediastinic dissection (mediastinic adipose and thymus).

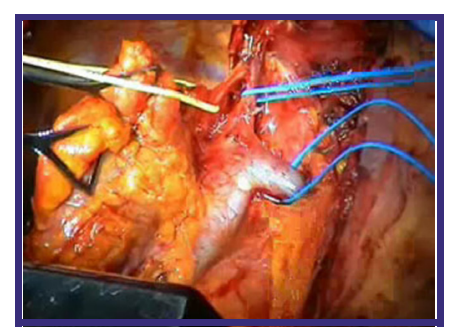

Video 4. (Patient B). In this case, the surgical approach chosen (hemi-clamshell) facilitated control of the innominate veins and separate control of the SVC, after opening the pericardium.

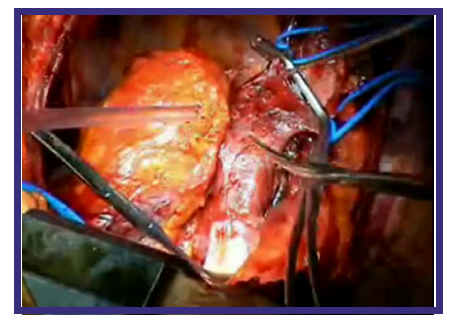

Video 5. (Patient B). Here the vena cava was cross-clamped as described previously without the use of extracorporeal circulation or a shunt. Heparin $(0.5 \mathrm{mg} / \mathrm{kg})$ was administered prior to clamping.

Table 1 summarizes postoperative mortality in recent published series. The recently-published multicentric study [10] on 109 patients operated on over a 40-year



Video 6. (Patient C). Same technique (cross clamping technique) as described in Video 5.

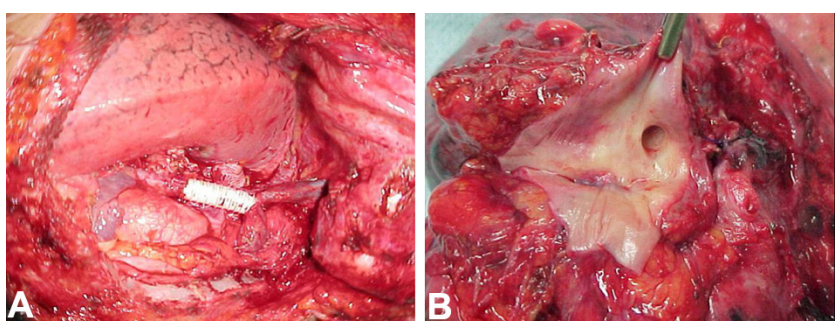

Photo 5. Complete SVC substitution. Hemi-clamshell approach; right superior lobectomy, reinforced PTFE prosthesis (size 14) for SVC-SVC anastomosis (A). (B) shows that the cancer has infiltrated the entire thickness of the vessel.

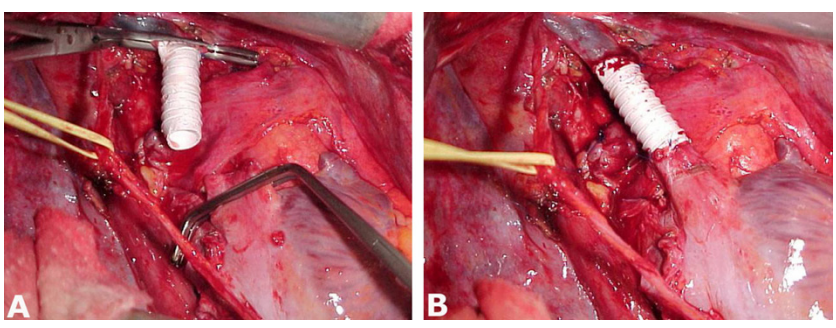

Photo 6. Complete SVC substitution. Approach: lateral thoracotomy; lung resection: tracheal sleeve pneumonectomy; ringed PTFE (size 14) prosthesis serves to achieve SVC-SVC anastomosis.

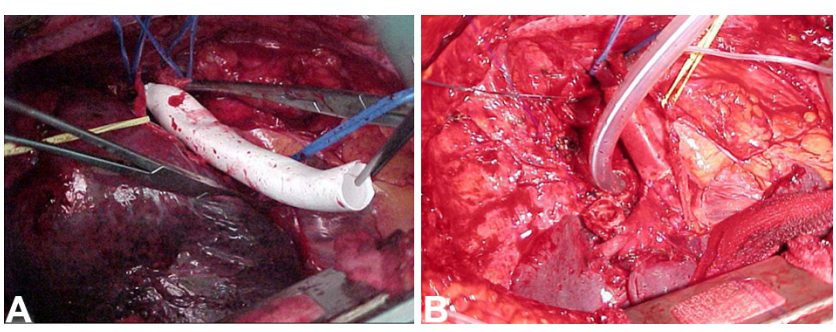

Photo 7. (A-B). Complete SVC substitution. Approach: lateral thoracotomy; lung resection: tracheal sleeve lobectomy with neocarina (B) SVC-SVC anastomosis with PTFE prosthesis (size 14).

period, reported intraoperative mortality at $0 \%$. Postoperative morbidity at $33 \%$ and postoperative mortality of $12 \%$. Respiratory complications $(60 \%)$ were responsible for $92 \%$ of postoperative deaths; $4.5 \%$ (5/109) of patients developed early SVC thrombosis: three of the $28(11 \%)$ who received complete resection with prosthetic substitution, and two of the 80 $(2.5 \%)$ who received partial SVC resection. Prosthesis infection occurred in $7 \%(2 / 28)$. 


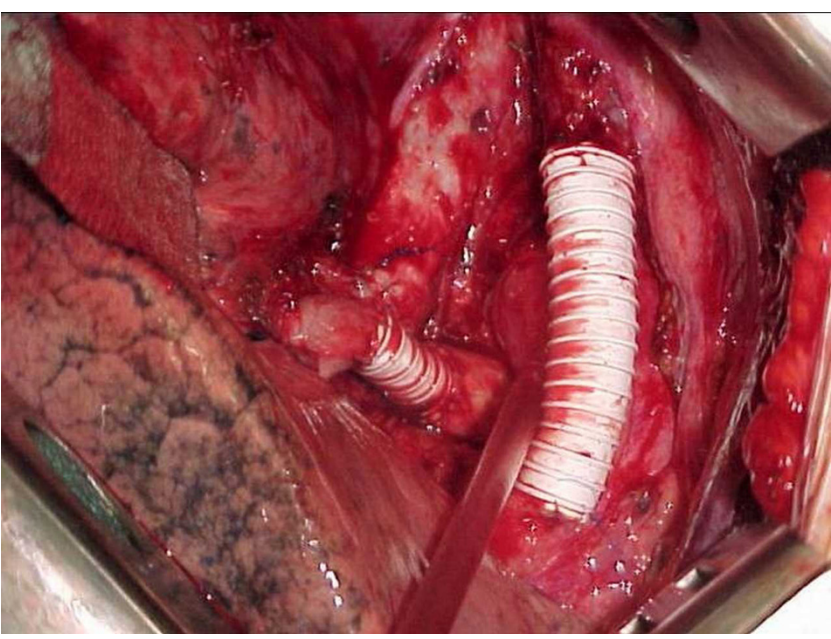

Photo 8. Complete SVC substitution. Both innominate veins resected for mediastinic tumour; approach: sternotomy; no lung resection; ringed PTFE (size 12) prosthesis for anastomosis between left innominate vein and SVC.

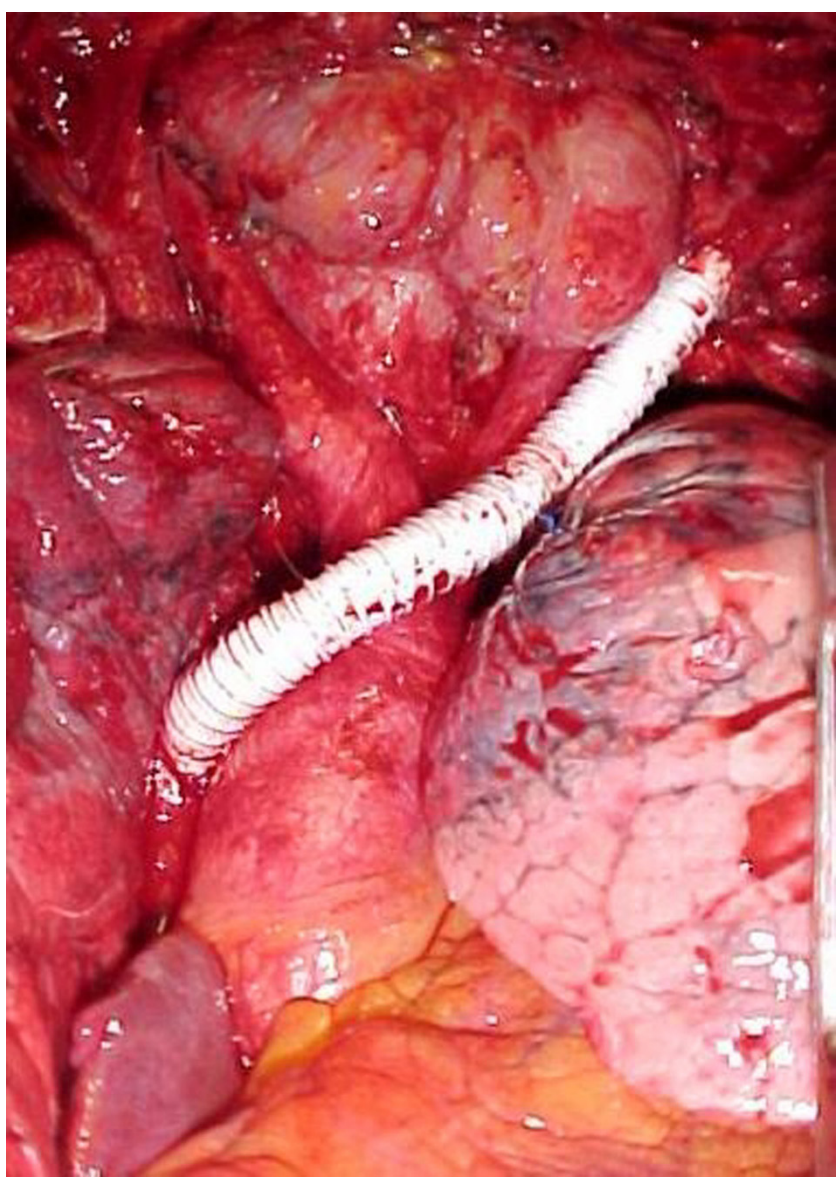

Photo 9. Complete SVC substitution. Approach: lateral thoracotomy; lung resection: superior right double sleeve lobectomy; ringed PTFE (n. 10) prosthesis for pulmonary artery; and ringed PTFE (size 12) prosthesis for SVC-SVC anastomosis.
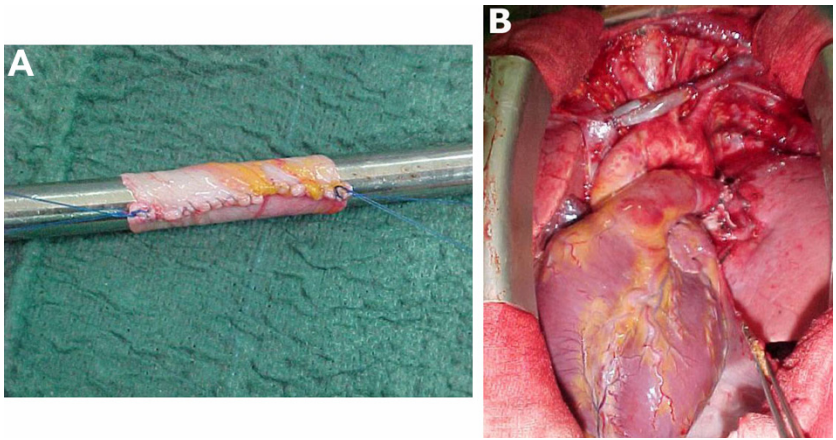

Photo 10. Left innominate vein substitution and partial SVC resection for mediastinic tumour. Approach: sternotomy; lung resection: left superior lobectomy with complete antero-superior mediastinectomy and pericardiectomy (B). Left innominate vein reconstructed with autologous pericardium (A).

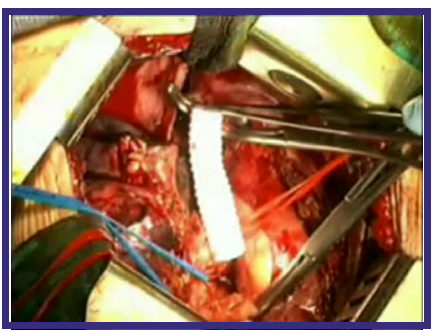

Video 7. (Patient C). Revascularisation of the SVC by ringed PTFE prosthesis.

Mean intensive stay period was three days and mean hospitalisation was 16 days. With regard to long-term outcomes, median survival was 11 months and 5-year survival probability was $21 \%$. Because the analysis covered a very long recruitment period, in which surgeons' experiences were variable and over which technology changed greatly, a separate analysis on patients operated on over the last 10 years was also presented. In this series postoperative mortality was $6 \%$ with 5 -year survival of $28 \%$.

Multivariate analysis identified performance of pneumonectomy as negatively influencing prognosis and type of SVC revascularisation as also independently influencing prognosis.

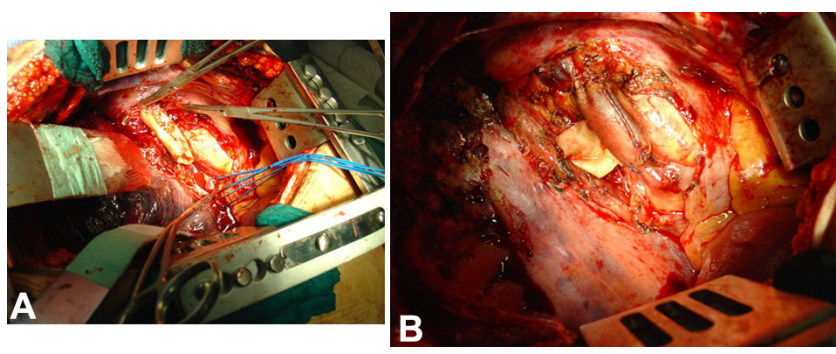

Photo 11. Complete SVC substitution for NSCLC. Approach: lateral thoracotomy; lung resection: tracheal sleeve. SVC reconstructed with heterologous pericardial graft, SVC-SVC anastomosis. 


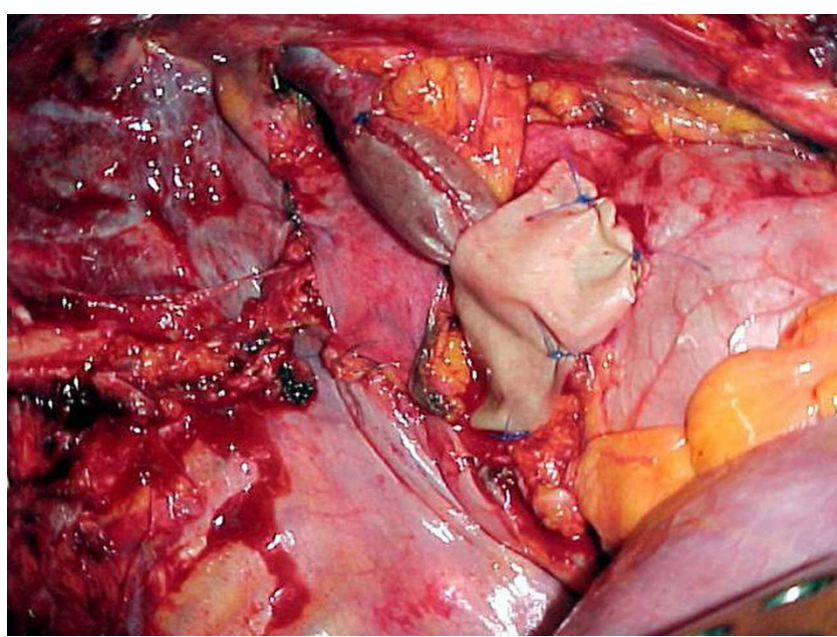

Photo 12. Similar case as Photo 11. Complete SVC substitution for NSCLC. Approach: lateral thoracotomy; lung resection: tracheal sleeve. SVC reconstructed with heterologous pericardial prosthesis, SVC-SVC anastomosis. Note the reconstruction of the pericardial defect with the same pericardial patch used for SVC prosthesis.

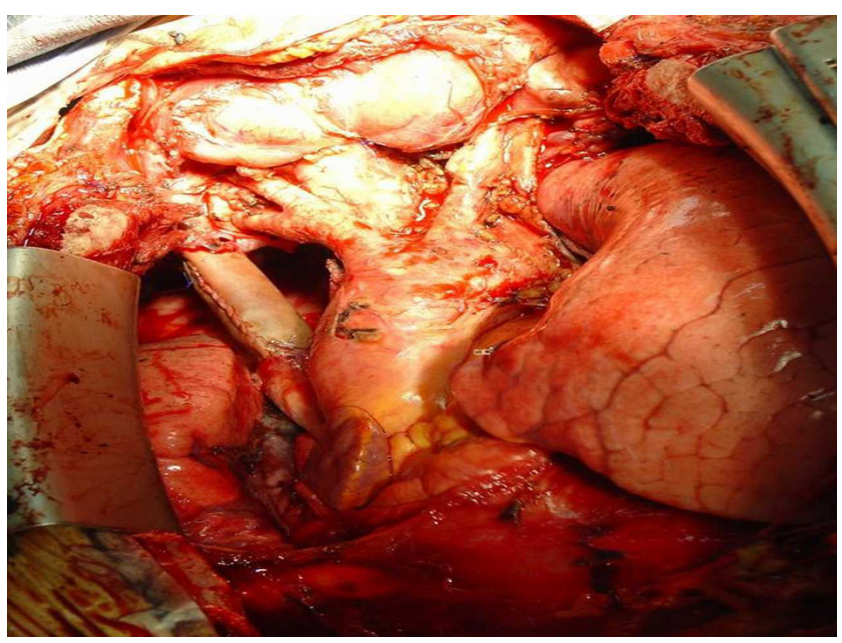

Photo 13. Complete SVC substitution with right and left innominate vein resection for invasive thymoma after chemo-radiation therapy. Approach: sternotomy. Lung resection: right upper lobectomy. SVC reconstruction with custom-made heterologous pericardial tube, between right innominate vein and SVC.

Our recent experience (personal communication, STS Chicago, 2006) consists of 70 patients who underwent SVC resection at the European Institute of Oncology from 1998 to 2004; 52 were operated on for NSCLS and 18 for mediastinic tumour. Lung cancer patients received induction chemotherapy in $77 \%$ of cases. Major postoperative morbidity and mortality were $23 \%$ and $7.7 \%$, respectively $(50 \%$ and $5.6 \%$ in mediastinal tumours). In the lung cancer group, median survival was 16.2 months $(95 \% \mathrm{Cl} 12.6-36.7)$ with 5 -year survival probability of $31 \%$. Median survival for mediastinal tumours was 49 months.

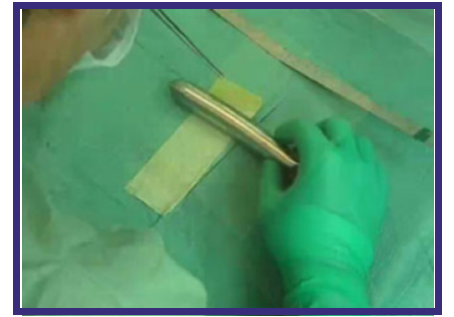

Video 8. (Patient A). The SVC was substituted with a prosthesis of bovine pericardium formed into a tube. The required $2 \mathrm{~cm}$ diameter was obtained with tutors and continuous polypropylene suturing $5 / 0$. Prosthesis length was close to that of the resected SVC.



Video 9. (Patient B). Same technique as Video 8.

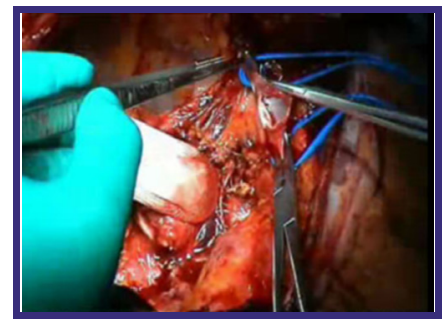

Video 10. (Patient B). The proximal anastomosis of the bovine custom-made prosthesis with the innominate veins confluence was constructed first using continuous polypropylene $5 / 0$ sutures, followed by the anastomosis with SVC. The lung resection was a right superior lobectomy.

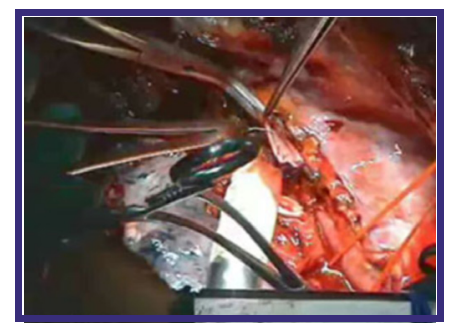

Video 11. (Patient A). The proximal anastomosis of the bovine custom-made prosthesis with the innominate veins confluence was constructed first using continuous polypropylene $5 / 0$ sutures, followed by the anastomosis with SVC. The lung resection was a tracheal sleeve pneumonectomy.

\section{Conclusions}

SVC infiltration implies at least locally advanced lung cancer for which combined chemotherapy and radio- 


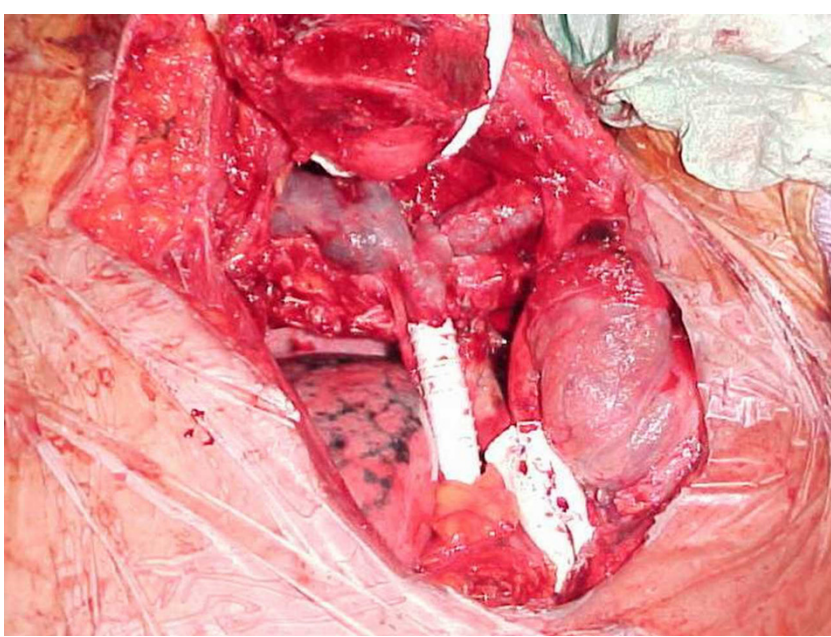

Photo 14. Complete SVC substitution with right and left innominate veins resection for NSCLC. Approach: transmanubrial and lateral thoracotomy. Lung resection: right superior lobectomy. SVC reconstructed with PTFE (size 12) prosthesis and anastomosis between right innominate vein and SVC.

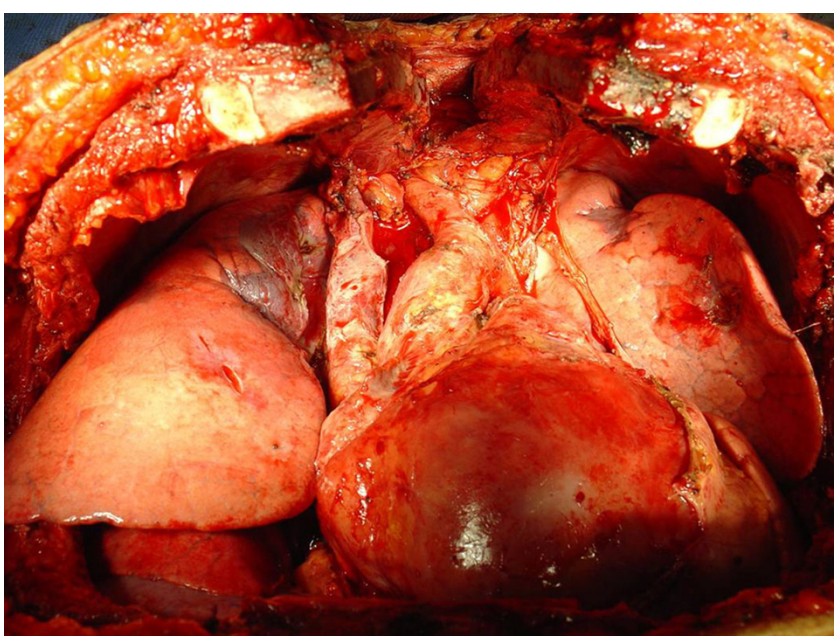

Photo 15. Germ cell cancer of mediastinum. Approach: clamshell and median sternotomy; partial resection of SVC and left innominate vein; no reconstruction.

therapy gives five year survival in the range 5\%-17\%. SVC resection is feasible: in that postoperative complications and mortality are acceptable using modern patient selection criteria. Patients with extensive nodal involvement (R2 nodes involved) or SVC infiltration from bulky N2, should not be candidates for SVC resection as oncological radicality will not be possible. Induction chemotherapy is advisable to identify patients with aggressive disease, and reduce the extent of resection.

Five year survival probability in all published studies is better than that obtained by chemotherapy/radiotherapy and is in the range $25 \%-31 \%$. The use of heterological prosthesis material, such as animal per-

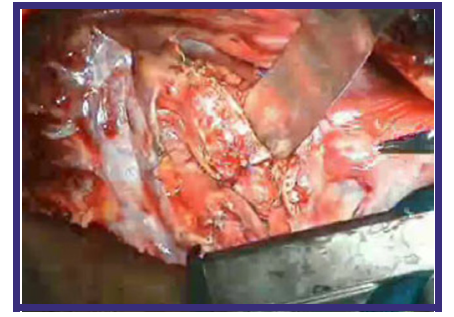

Video 12. (Patient A). In this case of SVC resection, tracheal sleeve pneumonectomy was necessary. After carinal resection, ventilation was achieved by intubation of the left main bronchus. The trachea was anastomosed with continuous double polypropylene sutures $3 / 0$. The bronchial anastomosis was separated from the caval prosthesis using a flap of intercostal muscle and a flap of vascularised mediastinic fat. The surgical specimen shows the carina en bloc with the right lung and the SVC.

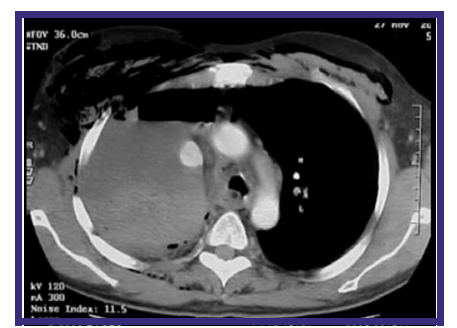

Video 13. (Patient A). Postoperative CT of the thorax, phlebography and $3 \mathrm{D}$ reconstruction of the airway.

icardium, promises to reduce the rate of infective and thrombotic postoperative complications.

\section{References}

[1] Ginsberg RJ, Vokes EE, Rosenzweig K. Non small cell lung cancer. In: DeVita VT, Hellman S, Rosenberg SA, editors. Cancer. Principles and practice of oncology 6th ed. Philadelphia: Lippincott-Raven, 2001:925-983.

[2] Piccione W, Penfield Faber L, Warren WH. Superior vena cava reconstruction using autologous pericardium. Ann Thorac Surg 1990; 50:417-419.

[3] Thomas P, Magnan PE, Moulin G, Giudicelli R, Fuentes P. Extended operation for lung cancer invading the superior vena cava. Eur J Cardiothorac Surg 1994;8:177-182.

[4] Tsuchiya R, Asamura H, Kondo H, Goya T, Naruke $T$. Extended resection of the left atrium, great vessels, or both for lung cancer. Ann Thorac Surg 1994;57:960-965.

[5] Dartevelle PG. Extended operations for the treatment of lung cancer. Ann Thorac Surg 1997;63:12-19.

[6] Fukuse T, Wada H, Hitomi S. Extended operation for non-small cell lung cancer invading great 


\section{Spaggiari et al. / Multimedia Manual of Cardiothoracic Surgery / doi:10.1510/mmcts.2005.001511}

vessels and left atrium. Eur J Cardiothorac Surg 1997;11:664-669.

[7] Spaggiari L, Regnard JF, Magdeleinat P, Jauffret B, Puyo P, Levasseur P. Extended resections for bronchogenic carcinoma invading the superior vena cava system. Ann Thorac Surg 2000;69: 233-236.

[8] Spaggiari L, Pastorino U. Combined tracheal sleeve and superior vena cava resection for nonsmall cell lung cancer. Ann Thorac Surg 2000;70:1172-1175.

[9] Bernard A, Bouchot O, Hagry O, Favre JP. Risk analysis and long-term survival in patients undergoing resection of T4 lung cancer. Eur $\mathrm{J}$ Cardiothorac Surg 2001;20:344-349.

[10] Spaggiari L, Magdeleinat $P$, Kondo $H$, Thomas $P$, Leon ME, Rollet G, Regnard JF, Tsuchiya R, Pastorino U. Results of superior vena cava resection for lung cancer. Analysis of prognostic factors. Lung Cancer 2004;44:339-346.

[11] Shargall Y, de Perrot M, Keshavjee S, Darling G, Ginsberg R, Johnston M, Pierre A, Waddell TK. 15 years single center experience with surgical resection of the superior vena cava for non-small cell lung cancer. Lung Cancer 2004;45:357-363.

[12] Suzuki K, Asamura H, Watanabe S, Tsuchiya R. Combined resection of superior vena cava for lung carcinoma: prognostic significance of patterns of superior vena cava invasion. Ann Thorac Surg 2004;78:1184-1189.

[13] Hunter W. The history of an aneurysm of the aorta with some remarks on aneurysms in general. Med Observ Inq 1757;1:323.

[14] Stokes W. A treatise on the diagnosis and treatment of diseases of the chest. I Diseases of the lung and windpipe. Dublin: Hodges Smith, 1837:370.

[15] Mclntire FT, Sykes EM Jr. Obstruction of the superior vena cava: a review of the literature and report of two personal cases. Ann Intern Med 1949;30:925-960.

[16] Carlson HA. Obstruction of the superior vena cava: an experimental study. Arch Surg 1934; 29:669.

[17] Klassen KP, Andrews NC, Curtis GM. Diagnosis and treatment of superior-vena-cava obstruction. AMA Arch Surg 1951;63:311-325.

[18] Bricker EM, McAfee CA. Femoral vein graft following bilateral internal jugular vein resection. Surgery 1952;32:114-118.

[19] Benvenuto R, Rodman FS, Gilmour J, Phillips AF, Callaghan JC. Composite venous graft for replacement of the superior vena cava. Arch Surg 1962;84:570-573.

[20] Schramel R, Olinde HDH. A new method of bypassing the obstructed vena cava. J Thorac Cardiovasc Surg 1961;41:375.

[21] Taylor GA, Miller HA, Standen JR, Harrison AW. Bypassing the obstructed superior vena cava with a subcutaneous long saphenous vein graft. J Thorac Cardiovasc Surg 1974;68:237240.

[22] Vincze K, Kulka F, Csorba L. Saphenous-jugular bypass as palliative therapy of superior vena cava syndrome caused by bronchial carcinoma. J Thorac Cardiovasc Surg 1982;83:272-277.

[23] Doty DB, Baker WH. Bypass of superior vena cava with spiral vein graft. Ann Thorac Surg 1976;22:490-493.

[24] Chiu CJ, Terzis J, MacRae ML. Replacement of superior vena cava with the spiral composite vein graft. A versatile technique. Ann Thorac Surg 1974;17:555-560.

[25] Mitchell IM, Saunders NR, Maher O, Lennox SC, Walker DR. Surgical treatment of idiopathic mediastinal fibrosis: report of five cases. Thorax 1986;41:210-214.

[26] Dartevelle P, Chapelier A, Navajas M, Levasseur P, Rojas A, Khalife J, Lafontaine E, Merlier M. Replacement of the superior vena cava with polytetrafluoroethylene grafts combined with resection of mediastinal-pulmonary malignant tumors. Report of thirteen cases. J Thorac Cardiovasc Surg 1987;94:361-366.

[27] Spaggiari L, Thomas $P$, Magdeleinat $P$, Kondo $H$, Rollet G, Regnard JF, Tsuchiya R, Pastorino U. Superior vena cava resection with prosthetic replacement for non-small cell lung cancer: longterm results of a multicentric study. Eur $\mathrm{J}$ Cardiothorac Surg 2002;21:1080-1086. 